\title{
OPTIMIZATION OF BACOSIDE A LOADED SNEDDS USING D-OPTIMAL MIXTURE DESIGN FOR ENHANCEMENT INSOLUBILITY AND BIOAVAILABILITY
}

\author{
MUKESH GOHEL ${ }^{a}$, ANKITA PUROHIT a, ASHA PATEL ${ }^{b}$, LAL HINGORANIc \\ aAnand Pharmacy College, Anand, Gujarat, India, bParul Institute of Pharmacy and Research, Vadodara, Gujarat, India, cPharmanza Herbal \\ Pvt. Ltd, Dharmaj, Gujarat, India \\ Email: ashaben.patel@paruluniversity.ac.in
}

Received: 15 Jun 2016 Revised and Accepted: 18 0ct 2016

\section{ABSTRACT}

Objective: The objective of present study is to enhance solubility and bioavailability of poorly soluble bacoside A present in Bacopa monnieri extract using self nano emulsifying drug delivery system (SNEDDS) for the treatment of Alzheimer's disease.

Methods: Solubility of the drug was assessed in various oils (edible as well as synthetic oil), surfactant and co-surfactant by saturation solubility study. Pseudo-ternary phase diagram was used to obtain appropriate concentration ranges of components include oil, surfactant and co-surfactant.

Results: From the result of saturated solubility study and phase diagram, oleic acid, tween 20 and ethanol was selected as oil, surfactant and cosurfactant. The D-Optimal mixture design was used to optimize the formulation on the basis of solubility of drug and dilution potential. In vitro dissolution, study showed $89 \%$ of drug release from optimized SNEDDS formulation compared to untreated drug extract with $24 \%$ of drug release in $60 \mathrm{~min}$. Ex vivo diffusion study showed more than $90 \%$ of drug diffused from optimized SNEDDS formulation compared to pure extract.

Conclusion: In a nutshell, the developed SNEDDS formulation using the design of experimentation approach held great potential as a possible alternative to traditional oral formulations of poorly soluble Bacoside $A$ to improve solubility and bioavailability.

Keywords: Bacoside A, Solubility, D-optimal mixture design, Bioavailability.

(C) 2016 The Authors. Published by Innovare Academic Sciences Pvt Ltd. This is an open access article under the CC BY license (http://creativecommons.org/licenses/by/4. 0/) DOI: http://dx.doi.org/10.22159/ijpps.2016v8i12.13488

\section{INTRODUCTION}

Bacosides obtained from Brahmi (Bacopa monnierii) mainly used in brain disorder and memory-related disorder which is dose dependent Inhibitor of Acetylcholine esterase (AChe) [1]. The bacosides help to repair damaged neurons by adding muscle to the kinase, the protein involved in the synthesis of new neurons to replace the old ones. Depleted synaptic activity is thus restored, leading to augmented memory functions [2]. The pharmacological effects of Bacopa monnieri are attributed to the presence of a number of biologically active compounds, including alkaloids, saponins and sterols. The compounds responsible for the memory enhancing effects of Bacopa monnieri are triterpenoid saponins called "Bacosides." The major chemical entity is shown to be responsible for the memory-facilitating action of extract, bacoside A and $B[3,4]$. Herbal drugs have the problem of poor solubility and hence less bioavailability. To overcome it, various technological strategies are reported in the literature including micronization, solid dispersions or cyclodextrin complex formation and different technologies of drug delivery systems. Among various approaches, self-micro/nano-emulsifying drug delivery system has gained more attention due to enhanced oral bioavailability enabling a reduction in dose, more consistent temporal profiles of drug absorption, selective targeting of drug towards specific absorption window in GIT, and protection of drug from the hostile environment in gut. Compared to conventional metastable emulsions, SNEDDS is a thermodynamically stable formulation with high solubilization capacity for lipophilic drugs and also can be filled directly into soft or hard gelatin capsules for convenient oral administration. Application of design of experiments (DoE) is a systematic approach to determine the relationship between factors affecting a process and the output of that process. Development of SNEDDS involves rational selection of excipients, like oil, surfactant, cosurfactant. One of the current requirements in the dossier for ANDA submission is the development of product considering the concepts of quality by design (QbD). Hence, through an understanding of excipient influence on the performance of the product is essential. The use of design experiments assists formulator to define design space, which will be helpful to the manufacturer. In which D-optimal mixture design technique provides an efficient means of optimizing the optimal formulation of a specific mixture of oil, surfactant, co-surfactant in self-micro emulsifying drug delivery system. The aim of the present study is to optimize the percentual composition, characterize and evaluate SNEDDS formulation of Bacopa monnieri extract employing the concept of design of experiment and enhancement in the solubility and bioavailability of bacoside $\mathrm{A}$.

\section{MATERIALS AND METHODS}

\section{Materials}

Dried extract of Bacopa monnierii (45\%) was provided ex-gratis from Pharmanza Herbal Pvt. Ltd, Dharmaj, India. The lipid excipients like Labrafac PG, Labrafac Lipophilic WL 1349, Labrafil M 2125CS, Labrasol and Transcutol HP were received from Gattefosse, Saint-Priest, France. Capmul MCM, Captex 200, Capmul 300 and Acconon were obtained from Abitec Corp., Columbus, OH. Tween 80, Tween 20 and Span 20, oleic acid, isopropyl myristate were obtained from SD fine Chemicals, Ahmadabad, India. Propylene glycol and PEG 400 were procured from Astron Chemicals, Ahmadabad, India. The buffer components, reagents were of analytical grade during the studies. The deionized double distilled water is used throughout the study.

\section{Methods}

Preliminary screening of components

\section{Screening of oil}

The solubility of Bacoside $A$ in various oils was determined by adding an excess amount of pure extract equivalent to Bacoside $A$ in $2 \mathrm{ml}$ of the oils separately in 5 -mL-capacity stopper vials, and mixed using a vortex mixer. The mixture vials were then kept at $25 \pm 1.0^{\circ} \mathrm{C}$ in an orbital shaker for 72 $\mathrm{h}$ to reach equilibrium. The equilibrated samples were removed from the shaker and centrifuged at 3,000 rpm for $15 \mathrm{~min}$. The supernatant was filtered through a $0.22-\mu \mathrm{m}$ membrane filter. The concentration of Bacoside $A$ was analyzed in oil by UV spectro-photometer [5].

\section{Screening of surfactant}

In water, $2.5 \mathrm{ml}$ of $15 \mathrm{wt}$. \% surfactant solution was prepared, and 4 
$\mu \mathrm{l}$ of oils were added with vigorous vortexing. If a one-phase clear solution obtained, the addition of the oil was repeated until the solution became cloudy. Selection of the surfactant was also carried out on the basis of oil solubilizing capacity of the surfactant as well as the solubility of the drug in surfactant.

\section{Screening of co-surfactant}

After the selection of the surfactant as described above selected surfactant was combined with six types of solubilizers as co-surfactants namely, ethanol, PEG 200, PEG 400, and propylene glycol in different ratio $1: 1,1: 2,1: 3,2: 1,3: 1$ and selection of the co-surfactant was done on the basis of clarity with surfactant. Clarity of different blends of $S_{\text {mix }}$ was determined by measuring \% Transmittance in Single beam U. V. Spectrometer. Selection of the co-surfactant also carried out on the basis of solubility of the drug in co-surfactant.

\section{Construction of pseudo-ternary phase diagram}

Pseudo-ternary phase diagrams were constructed in order to obtain the concentration range of components for the existing region of the microemulsion. Pseudo-ternary phase diagrams were constructed by Prosim software. Selected surfactant was blended with co-surfactant in the weight ratios of $4: 1,3: 1,2: 1,1: 1,1: 2$, and 1:3, Nine different combinations in different weight ratios of oil and $S_{\text {mix }}, 1: 9,2: 8,3: 7,4: 8$, $5: 5,6: 4,7: 3,8: 2$ and 9:1, were taken. One axis of the pseudo threecomponent phase diagram represented the aqueous phase, the other represented the oil phase, and the third represented a mixture of surfactant and co-surfactant at a fixed weight ratio $\left(S_{\text {mix }}\right)$.
IR spectroscopy was conducted using an FTIR spectrophotometer and the spectrum was recorded in the wavelength region of 4000$400 \mathrm{~cm}^{-1}$. [6] The procedure consisted of dispersing the samples in $\mathrm{KBr}$, thus avoiding solid transition possibly inducing by extended grinding. The spectrum was scan at a resolution of $0.15 \mathrm{~cm}^{-1}$ and scan speed $20 \mathrm{scan} / \mathrm{sec}$. The Infra-Red spectra of pure Bacopa monnieri extract and mixture with Oleic acid, Tween 20, Ethanol were obtained on Fourier Transform Infrared Spectrophotometer in order to detect the existence of an interaction between drug and excipients.

\section{Optimization of Bacoside A SNEDDS using D-optimal mixture design}

D-optimal mixture design was selected because the generalized variance of the estimates of the coefficients is minimized. The software selected a set of candidate points as a base design included factorial points (high and low level from the constraints on each factor, centers of edges, constraint plane centroids, axial checkpoint, and an overall center point). Moreover, the numbers of trials required are less. Eleven runs were carried out to optimize SNEDDS formulation. The effect of three formulation variables (amount of oils, surfactant, and cosurfactant) was studied on the solubility of drug $(\mathrm{mg} / \mathrm{ml})$ and nepheloid turbidity index [NTU] and viscosity. The optimum formulation of this study is selected to have a maximum solubility and minimum NTU. This design was generated second order polynomial equations which explained the non-linear nature of the response. Results of that statistical analysis were taken as significant if their corresponding $p$-values were $\leq 0.05$ [7].

\section{Drug excipient compatibility}

Table 1: Factors and levels with coded and transformed value

\begin{tabular}{lllll}
\hline \multirow{2}{*}{ Factors } & \multicolumn{2}{l}{ Levels } & Coded value & \\
\cline { 2 - 5 } & Transformed value & & High level & Low level \\
\cline { 2 - 5 } & High level & 2 & 1 & 0 \\
\hline X1(Oleic acid) & 18 & 15 & 1 & 0 \\
X2(Tween 20) & 36 & 5 & 1 & 0 \\
X3(Ethanol) & 12 & & Low level & \\
\hline
\end{tabular}

Table 2: Design matrix generated by design expert software (8.0.7.1)

\begin{tabular}{llll}
\hline Formulation code & Oil & Surfactant & Co-surfactant \\
\hline 1 & 18 & 15 & 12 \\
2 & 4.52 & 35.47 & 5 \\
3 & 6.094 & 26.906 & 12 \\
4 & 10.44 & 25.27 & 9.27 \\
5 & 10.44 & 25.27 & 9.27 \\
6 & 8.93 & 30.72 & 5.34 \\
7 & 12.098 & 20.90 & 12 \\
8 & 13.71 & 26.29 & 5 \\
9 & 2 & 31 & 12 \\
10 & 16.52 & 19.23 & 9.24 \\
11 & 18 & 22 & 5 \\
12 & 2 & 34.96 & 8.04 \\
\hline
\end{tabular}

\section{Characterization and evaluation of SNEDDS}

\section{Refractive index and percent transmittance}

Refractive index and percentage transmittance proved the transparency of formulation. The refractive index of the system was measured by Digital Abbe refractometer, Rochester by placing one drop of the formulation on the slide at $25{ }^{\circ} \mathrm{C}$ in triplicate and compared it with water [8-9].

\section{Self-emulsification time}

To determine emulsification time, $0.5 \mathrm{ml}$ of the SNEDDS formulation is mixed with $250 \mathrm{ml}$ of $0.1 \mathrm{~N} \mathrm{HCl}$ in a beaker by using a magnetic stirrer rotating at constant speed. Emulsification is done at room temperature, and the surface of the emulsion is examined for turbidity [10].

\section{Droplet size analysis and zeta potential measurement}

The droplet size of the emulsions was determined by photon correlation spectroscopy (which analyzes the fluctuations in light scattering due to the Brownian motion of the particles) using a zeta sizer able to measure sizes between 10 and $5000 \mathrm{~nm}$. The measurements were performed at $25^{\circ} \mathrm{C}$ at a $90^{\circ}$ angle. The formulation was dispersed into $100 \mathrm{ml}$ of water under gentle stirring in a glass beaker. Then a $1 \mathrm{ml}$ of the aliquot was withdrawn and added into a sample cell for droplet size measurement. Each size value reported was the average of at least three independent measurements. [11-12].

\section{Cloud point determination}

The optimized SNEDDS formulation was diluted with distilled water in the ratio of 1:250. The diluted sample was placed in a temperature regulated water bath, and the temperature was increased gradually. 
Cloud point was determined as the temperature at which there was a sudden appearance of cloudiness [13].

\section{Transmission electron microscopy}

The morphology of drug loaded SNEDDS was performed using (Technai G $\mathrm{G}^{2}$ Ultra twin FEI, Netherland). A drop of sample was placed onto a carbon coated grid to form a thin liquid film. The excess solution was removed and the sample was examined photographed at an accelerating voltage of $120 \mathrm{KV}$.

\section{In vitro dissolution studies}

The quantitative in vitro dissolution studies were carried out to assess drug release from oil phase into the aqueous phase by USP type II dissolution apparatus. $0.1 \mathrm{~N} \mathrm{HCL}(500 \mathrm{ml})$ is used as dissolution medium. The apparatus rotates at $50 \mathrm{rpm}$ with a temperature of $37 \pm 0.5^{\circ} \mathrm{C}$. Aliquots of $5 \mathrm{ml}$ were withdrawn at $0,10,20,30,40,50,60$ min, filtered through $0.45 \mu \mathrm{m}$ membrane filters and volume was withdrawn is replaced with fresh medium. Samples taken are then analyzed by using UV spectrophotometer.

\section{Ex vivo intestinal permeability study}

Male Wistar albino rats weighing (250-300 gm, 12-14 w age) were housed in cages with free access to standard diet water was given ad libitum. The rats were acclimatized to surrounding for one week prior to experiment were housed in cages with free access to standard diet water was given ad libitum. The experimental protocol was approved by Institutional Animal Experiment Ethics Committee of Anand Pharmacy College, Anand, Gujarat, India as per the guidance of CPCSEA, Ministry of Social Justice and Experiment, Government of India with approved protocol No. 1409/APC/2014-15. The animals were sacrificed by ether inhalation method. Intestine was isolated and cleaned properly and reconstituted SNEDDS, and the untreated extract was filled into intestine which was tied at both the end. The tissue was placed into tyrode solution with continuous aeration at 37 ${ }^{\circ} \mathrm{C}$ [14]. The beaker was filled by phosphate buffer solution $\mathrm{pH} 7.4$ with $1 \%$ SLS. At predetermined time intervals, samples were withdrawn from the beaker, and fresh buffer was replenished every time. The samples were analyzed spectrophotometrically for the content of drug. The \% diffusion was calculated, and the graph was plotted as absorption Vs time.

\section{RESULTS AND DISCUSSION}

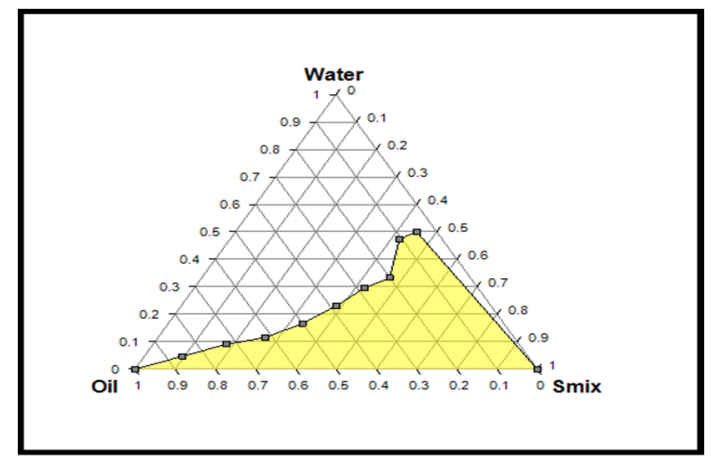

$(1: 1)$

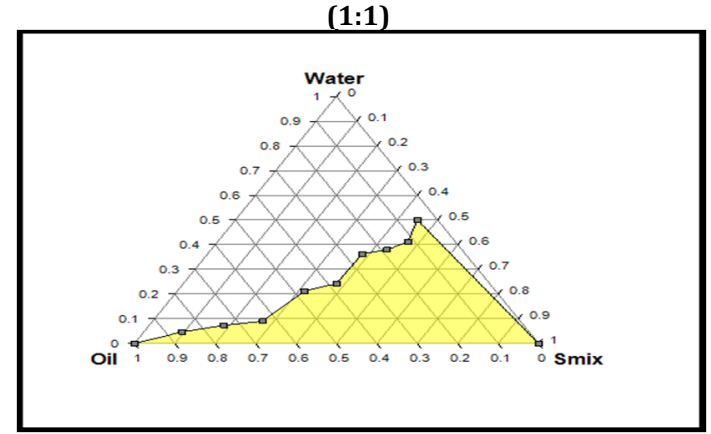

(2:1)

\section{Preliminary screening of components}

\section{Solubility study}

Amongst various oils tested, Bacoside $A$ showed low solubility in various oils except for oleic acid $(4.97 \pm 0.09 \mathrm{mg} / 2 \mathrm{ml})$ and turmeric oil for $45 \%$ of Bacoside $A$ in pure extract because Oleic acid is a monounsaturated omega-9 fatty acid, abbreviated with a lipid number of 18:1 cis-9. It has the formula $\mathrm{CH}_{3}\left(\mathrm{CH}_{2}\right) 7 \mathrm{CH}=\mathrm{CH}\left(\mathrm{CH}_{2}\right) 7 \mathrm{COOH}$.[14]. After selection of Oleic acid as the oil phase, the goal was to identify the surfactant which shows the highest solubilization capacity for the oil. Bacoside A shown maximum solubility in Tween $20(1.94 \pm 0.12 \mathrm{mg} / 2$ $\mathrm{ml}$ ) for $45 \%$ Bacoside A in Bacopa monnieri extract. Therefore, Tween 20 was selected as the surfactant for SNEDDS formulation. Amongst cosurfactant tested, maximum solubility in ethanol $(2.38 \pm 0.03 \mathrm{mg} / 2 \mathrm{ml})$ for $45 \%$ bacoside A in Bacopa monnieri extract. Therefore, Ethanol was selected as the co-surfactant for SNEDDS formulation. Whereas various cosurfactants were screened for solubility as well miscibility with a surfactant. Amongst various S mix blend, Ethanol forms transparent system $(99.87 \% \mathrm{~T})$ more than other tested cosurfactants with selected surfactant.

\section{Construction of pseudo-ternary phase diagram}

Initially, based on the results of maximum solubility, various pseudoternary phase diagrams were constructed employing Oleic acid (i.e. Oil), Tween 20 (i.e. surfactant) and ethanol (i.e. co-surfactant) for identifying the maximal region for formation of the thermo-dynamically stable nanoemulsion, as illustrated in fig. 1 . Among the various combinations of Tween 20 and ethanol (i.e. 1:1, 1:2, 1:3, 2:1, 3:1 and 4;1) explored, the maximal region for nanoemulsion was observed at the ratio of $3: 1$. An $\mathrm{o} / \mathrm{w}$ microemulsion region was found towards the water-rich apex of the phase diagram. As the surfactant concentration was increased in the $\mathrm{S}$ mix ratio, a higher microemulsion region was observed.

The probable reasons are a reduction of the interfacial tension, increased the fluidity of the interface. Kawakami $\mathrm{K}$ and coworkers reported that greater penetration of the oil phase occur in the hydrophobic region of the surfactant monomers [16] From pseudoternary phase diagrams, the formulations in which the amount of oil phase completely solubilized the drug and which could accommodate the optimum quantity of $S_{\text {mix }}$ and distilled water were selected for the optimization study.

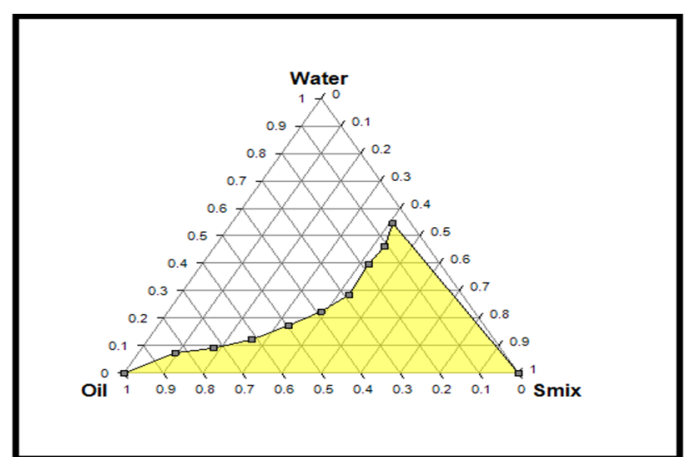

(1:2)

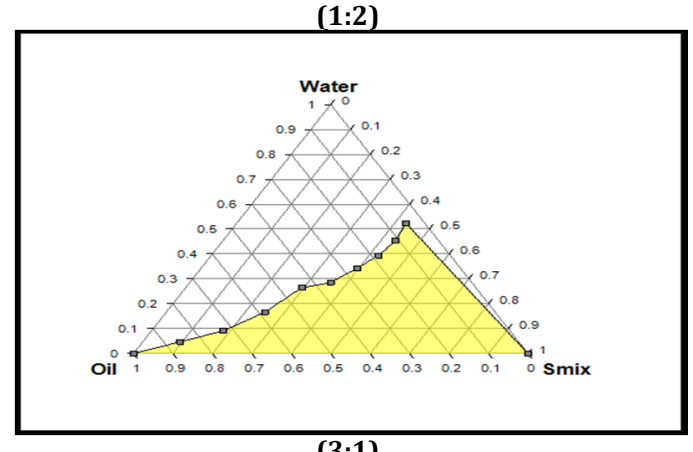

(3:1)

Fig. 1: Pseudo ternary diagrams of Oil: Smix (1:1), (1:2), (2:1), (3:1) 
Drug-excipient compatibility study

\section{FT-IR study}

\section{Compatibility study of Bacopa monnieri and excipients}

FT-IR studies were carried out for pure drug alone and along with excipients. The all characteristic peaks of pure extract were found in the mixture, suggested that there is no interaction between drug and excipients.
Optimization of Bacoside $A$ loaded SNEDDS using D-optimal mixture design

D-optimal mixture experimental design was applied in the present study to obtain optimum bacoside $A$ loaded SNEDDS. Oleic acid $\left(\mathrm{X}_{1}\right)$, Tween $20\left(\mathrm{X}_{2}\right)$, and Ethanol $\left(\mathrm{X}_{3}\right)$ were chosen as formulation variables, and Solubility of drug $(\mathrm{mg} / \mathrm{ml})\left(\mathrm{Y}_{1}\right), \mathrm{N}$. T. U (Nephlo turbidity unit) $\left(\mathrm{Y}_{2}\right)$ and Viscosity $(\mathrm{cp})\left(\mathrm{Y}_{3}\right)$ were selected as response variables. The responses of these formulations are summarized in table 3.

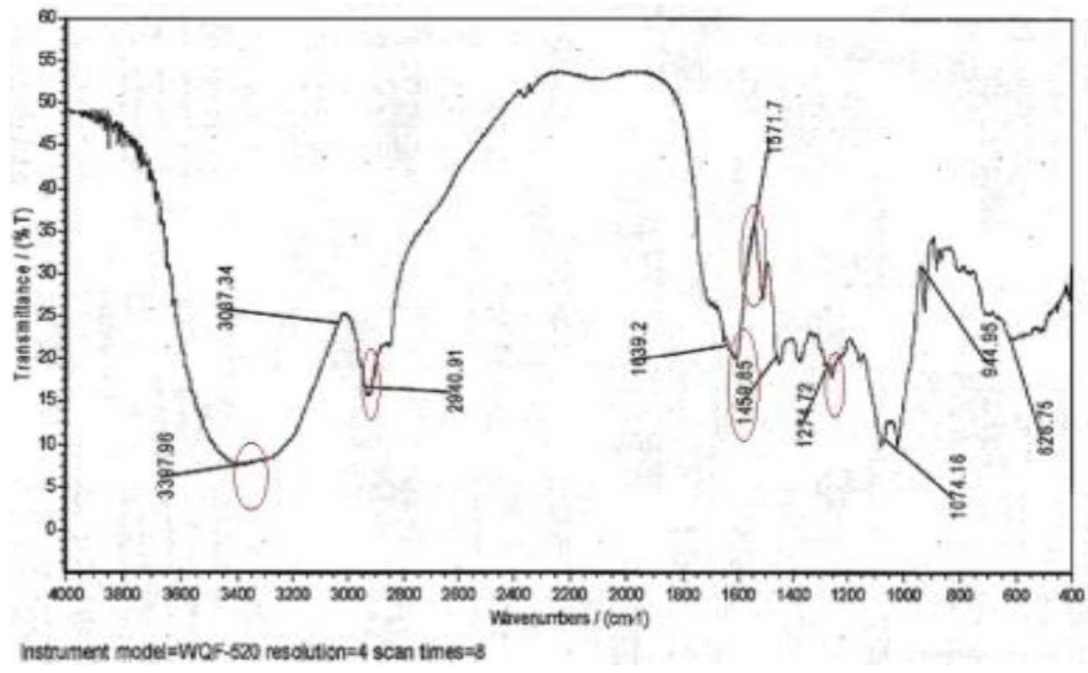

Fig. 2: FT-IR spectra of Bacopa monnieri

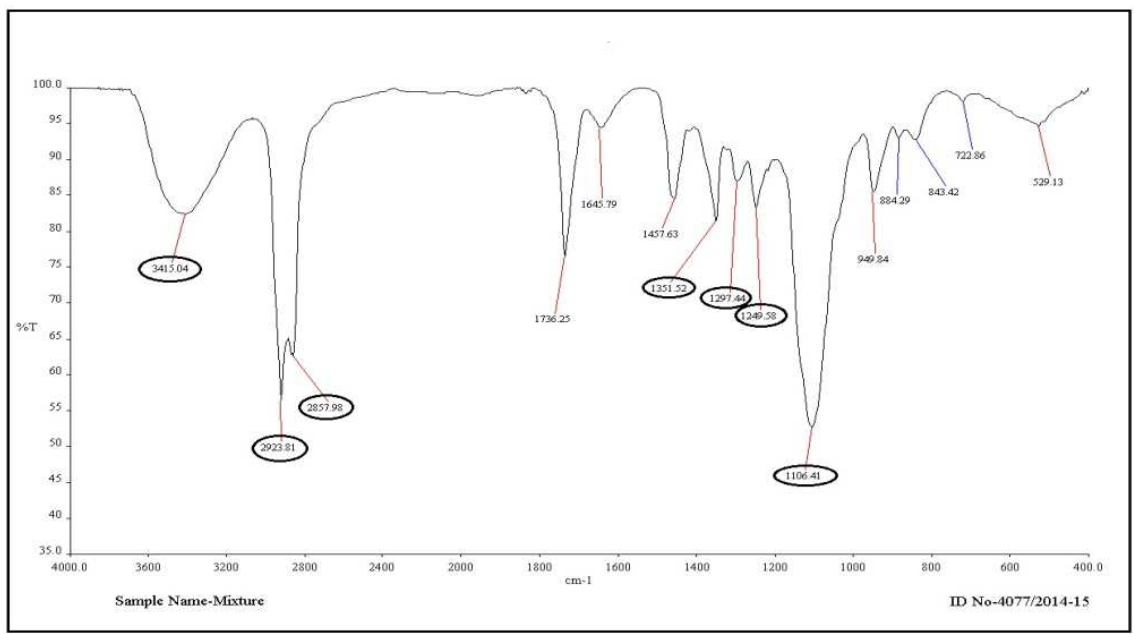

Fig. 3: FT-IR spectra of Bacopa monnieri+Excipient

Table 3: Responses of formulations as per D-optimal mixture design

\begin{tabular}{llll}
\hline Formulation code & Response & & \\
\cline { 2 - 4 } & Solubility $(\mathbf{m g} / \mathbf{m l})$ for 45\% bacoside A in pure extract & N. T. U (Dilution potential) & Viscosity(cp) \\
\hline 1 & $10.64 \pm 0.23$ & $104.97 \pm 0.25$ & $4.2 \pm 0.01$ \\
2 & $10.84 \pm 0.12$ & $5.16 \pm 0.12$ & $6.9 \pm 0.04$ \\
3 & $10.89 \pm 0.09$ & $2.08 \pm 0.06$ & $4.27 \pm 0.07$ \\
4 & $10.03 \pm 0.13$ & $91.71 \pm 0.21$ & $7.32 \pm 0.02$ \\
5 & $10.24 \pm 0.21$ & $93.25 \pm 0.29$ & $7.3 \pm 0.05$ \\
6 & $9.44 \pm 0.27$ & $60.82 \pm 0.15$ & $8 \pm 0.01$ \\
7 & $10.21 \pm 0.14$ & $61.80 \pm 0.32$ & $4.5 \pm 0.08$ \\
8 & $9.24 \pm 0.43$ & $60.54 \pm 0.05$ & $7.52 \pm 0.01$ \\
9 & $10.89 \pm 0.05$ & $3.36 \pm 0.09$ & $6.4 \pm 0.04$ \\
10 & $9.13 \pm 0.31$ & $84.58 \pm 0.11$ & $5.56 \pm 0.03$ \\
11 & $9.93 \pm 0.36$ & $78.09 \pm 0.19$ & $6.24 \pm 0.09$ \\
12 & $10.83 \pm 0.02$ & $4.16 \pm 0.03$ & $7.4 \pm 0.07$ \\
\hline
\end{tabular}

Data expressed were of mean \pm SEM $(n=3)$ 
The data obtained from solubility (response $\mathrm{Y}_{1}$ ), N. T. U (response $\mathrm{Y}_{2}$ ) Viscosity (response $\mathrm{Y}_{3}$ ) was analyzed using Design Expert ${ }^{\circledR}$ Software.
The coefficient of a cubic model of the independent variables is shown in table. 4.

Table 4: Coefficient of quadratic equation for each independent variables

\begin{tabular}{llll}
\hline Co-efficient & Solubility $(\mathbf{m g} / \mathbf{m l})$ & N. T. U & Viscosity \\
\hline $\mathrm{B} 1\left(\mathrm{X}_{1}\right)$ & +14.26 & +105.44 & +4.21 \\
$\mathrm{~B} 2\left(\mathrm{X}_{2}\right)$ & -1.22 & +9071.03 & +309 \\
$\mathrm{~B} 3\left(\mathrm{X}_{3}\right)$ & +1400.33 & -65.48 & +9.5 \\
$\mathrm{~B} 12\left(\mathrm{X}_{1} \mathrm{X}_{2}\right)$ & +2.2 & -16249.82 & -507.33 \\
$\mathrm{~B} 13\left(\mathrm{X}_{1} \mathrm{X}_{3}\right)$ & -1372 & +33.59 & -9.2 \\
$\mathrm{~B} 23\left(\mathrm{X}_{2} \mathrm{X}_{3}\right)$ & +2.19 & -16385.38 & -554.94 \\
$\mathrm{~B} 123\left(\mathrm{X}_{1} \mathrm{X}_{2} \mathrm{X}_{3}\right)$ & -2.27 & +17748.12 & +624.03 \\
\hline
\end{tabular}

The mixture components and response variables were related using the polynomial equation with statistical analysis though Design-Expert $\mathbb{R}$ 7.0.1 software. The value of the coefficients exhibits the effect of these variables on the response. The polynomial equations comprise the coefficients for intercept, main first-order effects, interaction term. A positive sign of coefficient indicates a synergistic effect while negative term indicates an antagonistic effect on the response. After generating the polynomial equations through MLRA (Multiple linear regression analysis) relating the dependent and independent variables, mixture components were optimized for the responses $\mathrm{Y}_{1}$ and $\mathrm{Y}_{2}$.
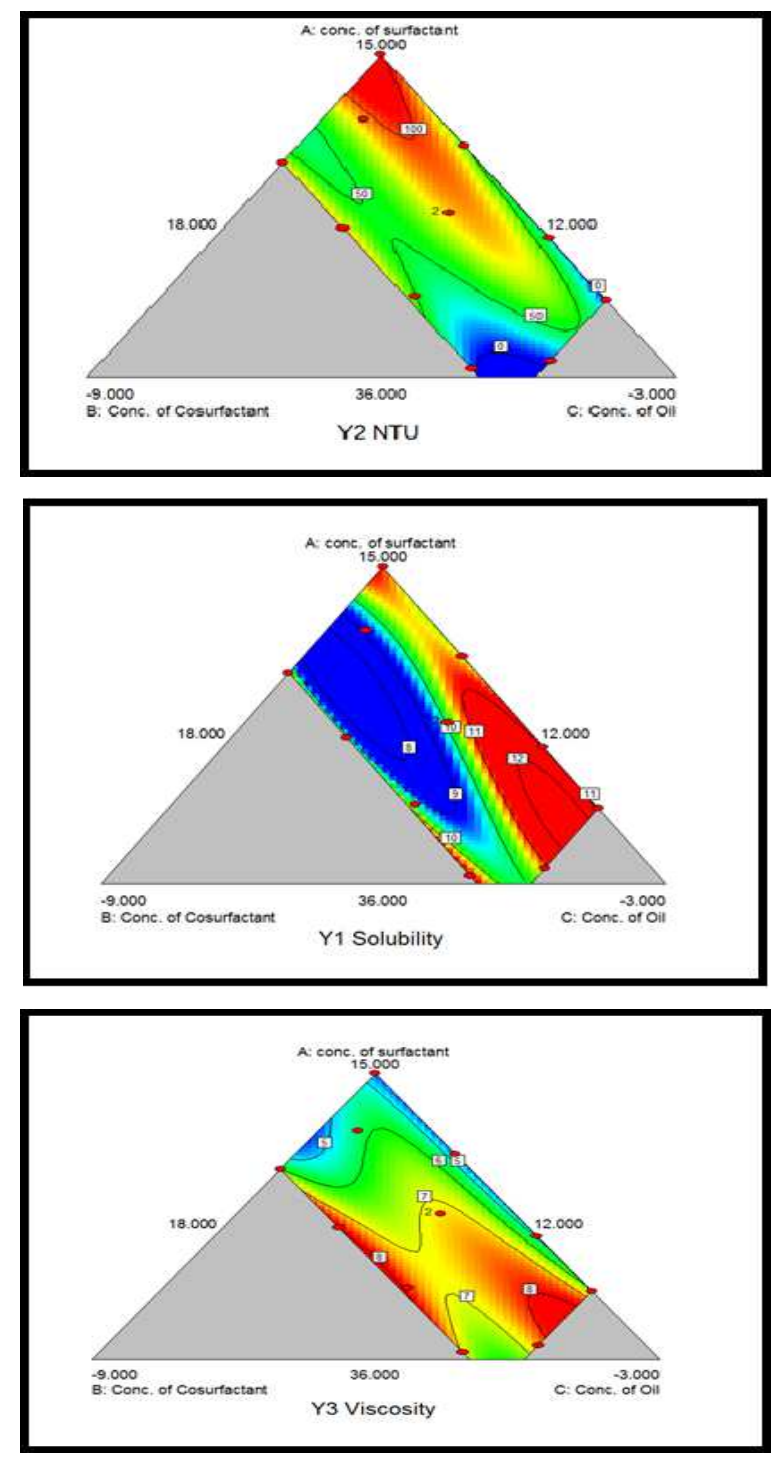

Fig. 4: Contour plots of response variables solubility, viscosity and NTU
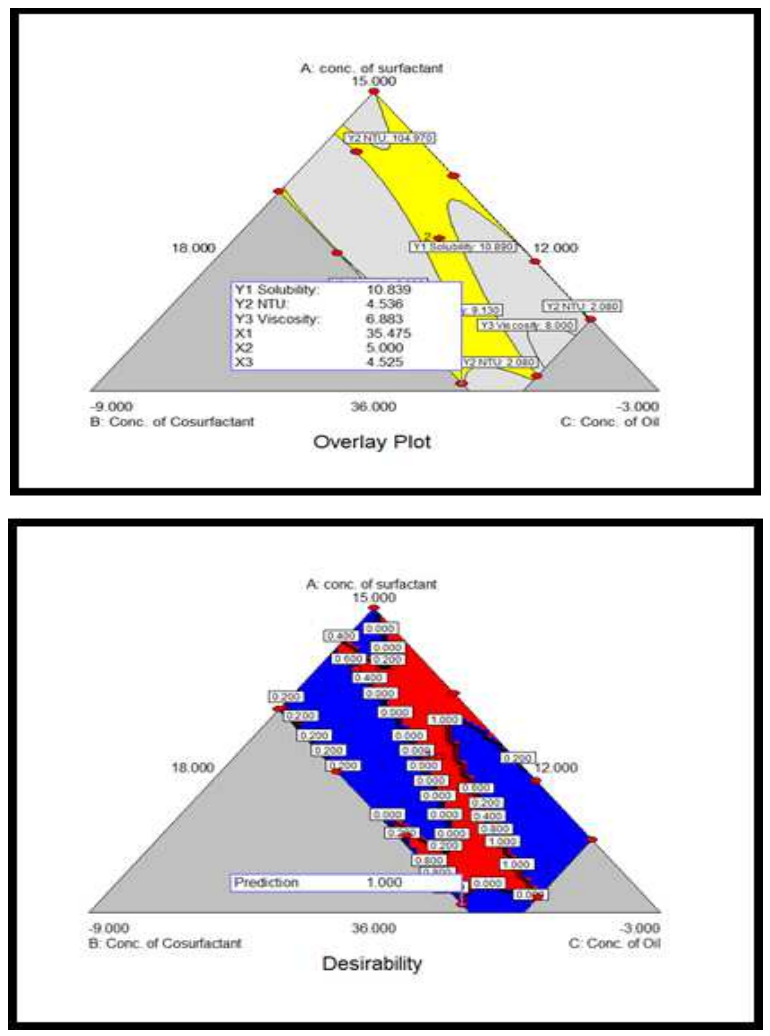

Fig. 5: (a) Overlay plot and (b) Desirability plot

Fig. 4 shows contour, 3D response, and overlaid plot. Overlapping of the contour plot should be done to select the design space. The overlaid area indicates the design space. The surfactant concentration should be kept as low as possible in the ideal formulation to avoid any associated toxicity.

The model was also fitted with a special cubic polynomial model and optimized by the "total subset" variable selection method after calculating with a step width of 0.1 , the maximum desirability function (0.97) value. To confirm the model adequacy for prediction, one batch of formulation under the optimum composition were prepared. The results shown in table 6 demonstrated good predictability of model.

Table 5: Optimized composition of formulation generated by design expert

\begin{tabular}{lll}
\hline Component & Amount in $\%$ w/w & Type \\
\hline Oleic acid & $4.52 \pm 0.12$ & Oil \\
Tween 20 & $35.47 \pm 0.36$ & Surfactant \\
Ethanol & $5.01 \pm 0.58$ & Co-surfactant \\
\hline
\end{tabular}

Data expressed were of mean \pm SEM $(n=3)$ 
Table 6: Checkpoint analysis of optimized batch

\begin{tabular}{lll}
\hline Parameters & Predicted value & Observed value \\
\hline Solubility $(\mathrm{mg} / \mathrm{ml})$ & $10.83 \pm 2.15$ & $10.65 \pm 1.25$ \\
NTU & $4.53 \pm 1.02$ & $4.28 \pm 2.35$ \\
Viscosity $(\mathrm{cp})$ & $6.88 \pm 1.08$ & $6.47 \pm 1.17$ \\
\hline
\end{tabular}

Data expressed were of mean \pm SEM (n=3), Bias (\%) = (predicted value - experimental value) $/$ experimental value $\times 100$.

\section{Characterization and evaluation of SNEDDS of Bacoside A}

\section{Refractive index and percent transmittance}

Transparency of formulation was determined in the termed of Transmittance $(\% \mathrm{~T})$ and Refractive index. The refractive index is the net value of the components of the microemulsion and indicates the isotropic nature of the formulation. The refractive index of optimized SNEDDS formulation was found to be 1.33 indicated transparency nearer to water (1.33) refer clear and isotropic in nature. Percentage transmittance of optimized SNEDDS was found to be $(98.7 \pm 0.08)$, and these results indicated the high clarity of microemulsion [15].

\section{Self-emulsification time}

Emulsification time was the parameter used for the assessment of the efficiency of emulsification which should disperse completely and quickly when subjected to aqueous dilution under mild agitation. A time of two minutes was used the ideal evaluation index in the emulsification process. The process of self-emulsification must occur with minimal agitation rather than require a negative free energy. [17] It was reported that when a self-emulsified system is diluted by the aqueous phase, various mesomorphic phases are observed between the formulation and water. A delay in the emulsification time with decreasing $\mathrm{S}_{\text {mix }}$ content may be because of the time required for the transformation from one liquid crystalline structure to another during the emulsification process. Self-emulsification time for optimized SNEDDS formulation was found to be 9 sec [18]

\section{Droplet size analysis and zeta potential measurement}

The droplet size of the emulsion is an important factor in selfemulsification performance because it determines the rate and extent of drug release as well as drug absorption. It has been reported that smaller the droplet size of the emulsion, higher the absorption and improved bioavailability. Decrease in particle size may be the result of non-ionic surfactants being available to stabilize the oil-water interface. Furthermore, the decrease in the droplet size behavior reflects the formation of a better closed packed film of the surfactant at the oil-water interface, thereby stabilizing the oil droplets. The smaller the droplet size, the larger the interfacial surface area will be provided for drug absorption. It appeared that the particle size was inversely proportional to the concentration of oil and directly proportional to the concentration of S mix. [19]. The Smaller particle size of SNEDDS may impart faster release of the drug. The optimized batch has a mean particle size of $33.84 \mathrm{~nm}$ in water.

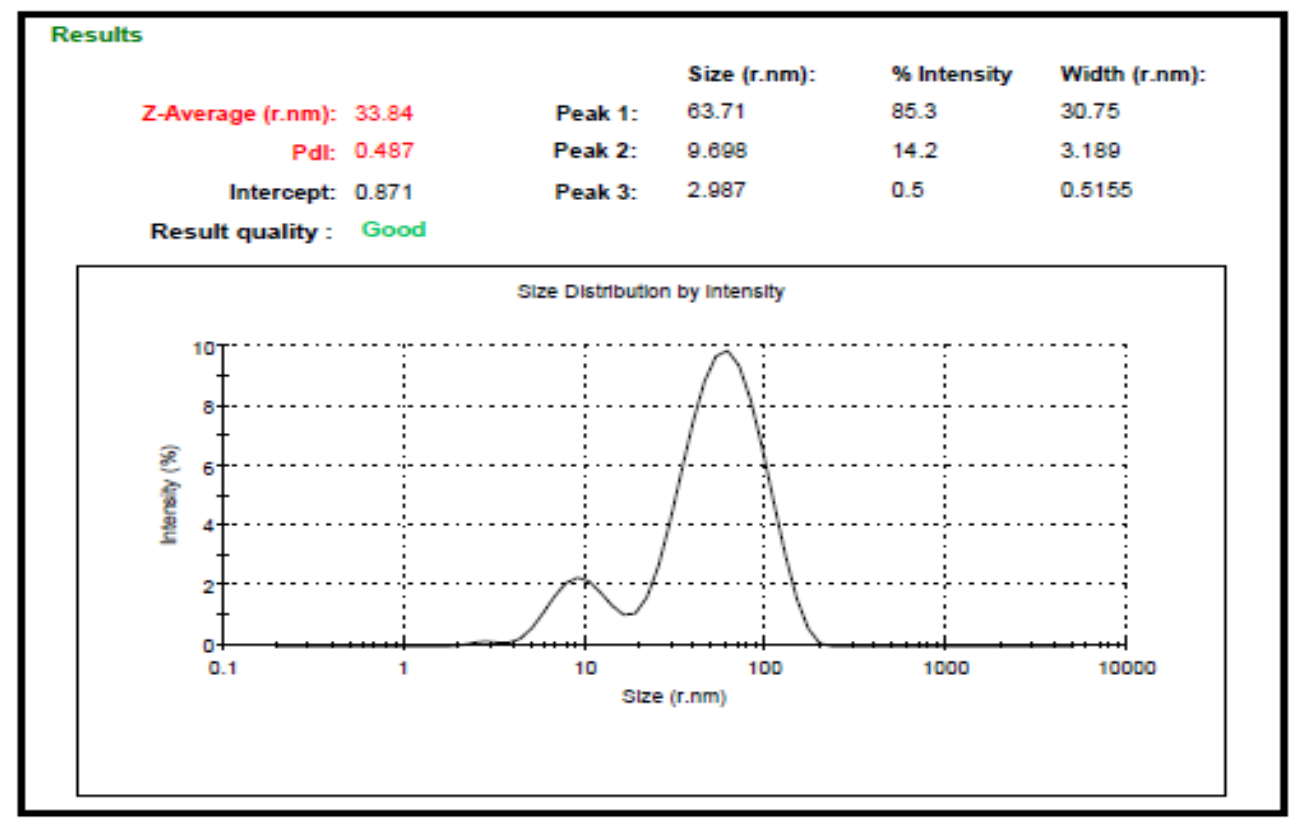

Fig. 6: (a) Graph of particle size measurement

The zeta potential governs the stability of microemulsion; it is important to measure its value for stability samples. The high value of zeta potential indicates electrostatic repulsion between two droplets. According to DLVO theory, +electric double layer repulsion will stabilize microemulsion where electrolyte concentration in the continuous phase is less than a certain value. A negative force means a negative potential between the droplets [18]. A novel SNEDDS, which results in the negatively charged dispersed oil droplets upon dilutions with an aqueous phase, leads to adhesion to the intestinal mucosa and thereafter drug uptake from the mucosa. Thus, these formulations enhanced the oral absorption and thus oral bioavailability. In this study, it was observed that zeta potential of the optimized batch was found to be $-4.45 \mathrm{mV}$. This result attributed due to the presence of non-ionic surfactant in SNEDDS.

\section{Transmission electron microscopy (TEM)}

When the samples were viewed under the electron microscope, discrete particles with an outer cubic structure could be viewed as presented in fig. 7. The particle size was found to be in the range of $6.12 \mathrm{~nm}-50 \mathrm{~nm}$. 


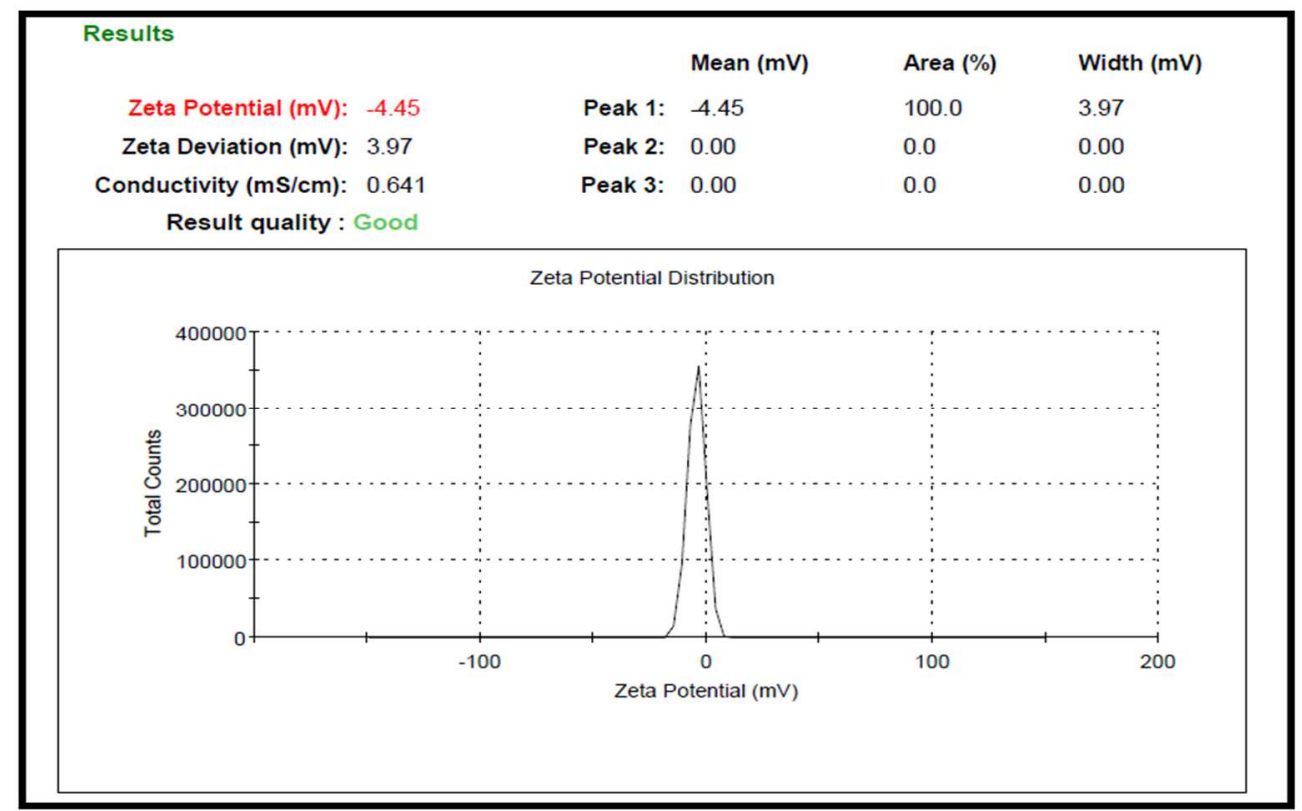

Fig. 6: (b) Graph of zeta potential measurement

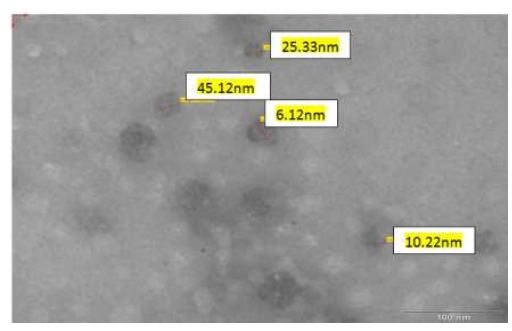

Fig. 7: Transmission electron microscopic image of optimized formulation

\section{In vitro dissolution study}

In this study, a comparison the dissolution profile of pure drug Bacoside A and SNEDDS formulation has been made. Optimized SNEDDS formulation shows more than $80 \%$ drug released from SNEDDS within $50 \mathrm{~min}$ where in the case of the unformulated bacoside A, up to $24 \%$ of the drug release within $50 \mathrm{~min}$. The faster dissolution from the optimized SNEDDS formulation can be attributed to the fact that, the drug is insolubilized form in the formulation and upon exposure to the dissolution medium, it results in the formation of smaller droplets size that can dissolve rapidly in the dissolution medium [18].

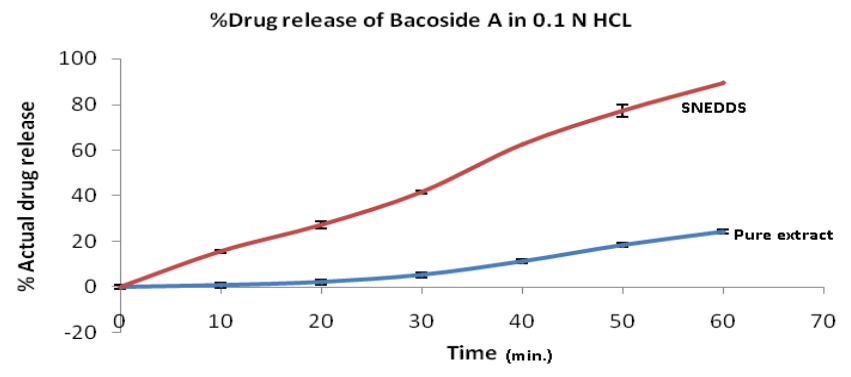

Fig. 8: In vitro dissolution profile of optimized SNEDDS formulation and untreated extract, Data expressed were of mean \pm SEM ( $n=6$ )

This result may be attributing to less emulsification time and droplet size of the emulsion, which permits faster drug release. Here, we have assumed a good correlation between Vitro and Vivo. Thus, we can say that the formulated NE improved the solubilization of BA and provided uniform in vitro release.

\section{Ex vivo intestinal permeability study}

Among all absorption screening methods, in situ intestinal perfusion study in rats is considered as a simple and relevant method of absorption assessment since the absorption properties are most similar to human beings. [19] The results of ex vivo intestinal permeability study were shown in fig. 9. After $4 \mathrm{~h}$ of diffusion, $90.14 \%$ of the drug was diffused from SNEDDS. While from the plain drug suspension, (pure untreated extract) the diffusion was found to be $23.65 \%$.

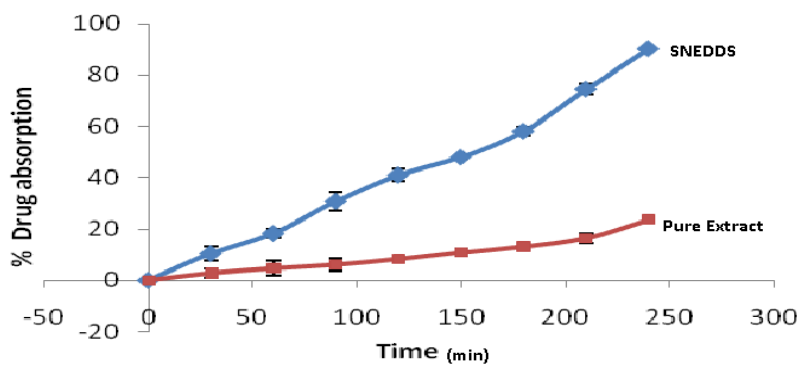

Fig. 9: Ex vivo intestinal permeability behavior of optimized SNEDDS and pure extract, Data expressed were of mean \pm SEM $(n=2)$ 
Bacoside A in the Nano emulsifying form could be absorbed completely at a faster rate in rat intestine than that in pure extract dispersion. Thus the amount of drug diffused through the biological membrane has more when it is given in the form of SNEDDS. Youenang Piemi et al. reported that the surface charge-modified droplets had a significant influence on the binding affinity of droplets to the skins, which could promote the bioavailability of drugs. [20] The enhancement in diffusion is due to the formation of microemulsion droplets in the nanometer range and improved the permeation of the Bacoside A because the presence of a surfactant, which reduces the interfacial tension of formulation.

\section{CONCLUSION}

The current research work involves the systematic development of optimized SNEDDS formulation of Bacoside A employing formulation by design approach and their subsequent evaluation using in vitro release studies, ex vivo permeation studies and in vivo studies for determining their enhanced bioavailability potential. Equilibrium solubility studies carried out in various oils, surfactant and Cosurfactant for rational optimization of the formulation using Doptimal mixture design. Bacoside A-loaded SNEDDS could spontaneously form small self-emulsifying droplets with an average droplet size of about $6.12 \mathrm{~nm}-50 \mathrm{~nm}$. Dissolution percentage of in $\mathrm{pH}$ 1.2 buffer solutions was significantly higher than Bacoside A thus, confirming distinct superiority in biopharmaceutical performance potential. The absorption of optimized formulation in rat intestine was via passive transfer by diffusion across the lipid membranes. The dose of Bacoside A used to treat could be decreased due to the high solubility of the drug in microemulsion inculcates significant therapeutic effect. Our studies proved a promising platform for improving solubility, intestinal permeability and bioavailability of active herbal principles with poor aqueous solubility.

\section{ACKNOWLEDGEMENT}

The authors are thankful to M/s Pharmanza Pvt. Ltd, Dharmaj, India, for providing the ex-gratis sample of Bacoside A. M/s Gattefosse, Saint-Priest, France; M/s BASF, Ludwigshafen, Germany, M/s Abitec Corp, Ohio, USA; and M/s M/s SD fine Chemicals, Ahmadabad, India, $\mathrm{M} / \mathrm{s}$ Astron Chemicals, Ahmadabad, India; are gratefully acknowledged and appreciated for providing the gift samples of excipient employed during the current study.

\section{CONFLICT OF INTERESTS}

There is no conflict of Interest

\section{REFERENCES}

1. Das A, Shanker G, Nath C, Pal R, Singh. A comparative study in rodents of standardized extracts of Bacopa monnierii and Ginkgo Biloba: anticholinesterase and cognitive enhancing activities. Pharmacol Biochem Behav 2002;73:893-900.

2. Tripathi Y. B, Bacopa monnierii Linn. As an antioxidant: mechanism of action. Indian J Exp Biol 1996;34:523-6.

3. Martis G, Rao A. Neuropharmacological activity of Herpestis monnierii. Fitoterapia 1992;63:399-404.

4. Russo A, Borrelli F. Bacopa monnieri, a reputed nootropic plant: an overview Elsevier; 2005. p. 305-17.

5. Azeem A, Rizwan M. Nanoemulsion components screening and selection, a technical note. AAPS PharmSciTech 2009;10:1-6.
6. Khaled Tahami. Preparation, characterization, and in vitro release of ketoprofen loaded alginate microspheres. Int J Appl Pharm 2014;6:9-12.

7. Pankaj Laddha, Vrunda Suthar, Shital Butani. Development and optimization of self-are micro emulsifying drug delivery of Domperidone. Braz J Pharm Sci 2014;50:90-100.

8. Sapra K, Saprab A, Singha SK. Self-emulsifying drug delivery system, a tool in solubility enhancement of poorly soluble drugs. Indo Glob J Pharm Sci 2012;2:313-32.

9. Sachan R, Khatri K, Kasture S. Self emulsifying drug delivery system approach for enhancement of bioavailability. Int J PharmTech Res 2010;2:1738-45.

10. Neeta Choudhary, DS Rathore. A review on self-emulsifying drug delivery system (SEDDS). Asian J Biochem Pharm Res 2014;4:109-18

11. Zhao Yi, Wang C, Chow A, Ke Ren, Gong T, Zhang Z, et al. Self nano-emulsifying drug delivery system for oral delivery of Zedoary essential oil, Formulation and bioavailability studies. Int J Pharm 2010;383:170-7.

12. Sierra A, Naveros B. Design, and optimization of self-nano emulsifying drug delivery systems for enhanced dissolution of gemfibrozil. Int J Pharm 2012;431:161-75.

13. Negi L, Tariq M, Talegaonkar S. Nanoscale self-emulsifying oil based carrier system for improved oral bioavailability of camptothecin derivative by P-Glycoprotein modulation. Colloids Surf B 2013;111:346-53.

14. Zhou H, Wan J, Wu L, Yi T, Liu W, Xu H, et al. A new strategy for enhancing the oral bioavailability of drugs with poor water solubility and low liposolubility based on phospholipid complex and supersaturated SEEDS. Int J Pharm Sci Res 2013;8:1-12.

15. Kawakami K, Yoshikawa T, Moroto Y, Kanaoka E, Takahashi K, Nishihara Y. Microemulsion formulation for enhanced absorption of poorly soluble drugs I. prescription design. J Controlled Release 2002;81:65-74.

16. Linjie L, Xiujuan $\mathrm{P}$, Wei Z, Siling W. Formulation design and in vitro evaluation of silymarin-loaded self-micro emulsifying drug delivery systems. Asian J Pharm Sci 2007;2:150-60.

17. Latika M Ingle, Vikrant $P$ Wankhade, Tapar K. New approaches for development and characterization of SNEDDS. Int J Pharm 2013;3:7-14.

18. $\mathrm{Mu} \mathrm{L}$, Feng SS. Fabrication, characterization and in vitro release of paclitaxel (Taxol) loaded poly (lactic-co-glycolic acid) microspheres prepared by spray drying technique with lipid/cholesterol emulsifiers. J Controlled Release 2001;76:239-54.

19. Shantha KTR, Chawla S, Nachaegari SK, Singh SK, Srinivas NR. Validated HPLC analytical method with programme wavelength UV detection for simultaneous determination of DRF 4367 and phenol red in rat in situ intestinal perfusion study. J Pharm Biomed Anal 2005;38:173-9.

20. Youenang Piemi MP, Korner D, Benita S, Marty JP. Positively and negatively charged submicron emulsions for enhanced topical delivery of antifungal drugs. J Controlled Release 1999;58:17787.

\section{How to cite this article}

- Mukesh Gohel, Ankita Patel, Asha Patel, Lal Hingorani. Optimization of Bacoside, a loaded SNEDDS, using D-optimal mixture design for enhancement in solubility and bioavailability. Int J Pharm Pharm Sci 2016;8(12):213-220. 